\title{
Assessment of the relative success of sporozoite inoculations in individuals exposed to moderate seasonal transmission
}

\author{
Adama Tall ${ }^{1}$, Cheikh Sokhna ${ }^{2}$, Ronald Perraut ${ }^{1}$, Didier Fontenille ${ }^{2}$, \\ Laurence Marrama ${ }^{1}$, Alioune B Ly ${ }^{1}$, Fatoumata D Sarr ${ }^{1}$, Aïssatou Toure ${ }^{1}$, Jean- \\ François Trape ${ }^{2}$, André Spiegel ${ }^{1}$, Christophe Rogier ${ }^{3}$ and Pierre Druilhe ${ }^{* 4}$
}

\begin{abstract}
Address: ${ }^{1}$ Unité d'Epidémiologie, Institut Pasteur de Dakar, B.P. 220 Dakar, Sénégal, ${ }^{2}$ UR 077 Paludologie Afrotropicale BP 1386, Dakar, Senegal ${ }^{3}$ URBEP, UMR 6236 - URMITE, IRBA, BP46, BP60109, 13262 Marseille, France and ${ }^{4}$ Bio-Medical parasitology Unit, Institut Pasteur de Paris, 28 rue Dr Roux, 75015, France

Email: Adama Tall - tall@pasteur.sn; Cheikh Sokhna - sokhna@ird.sn; Ronald Perraut - perrault@pasteur.sn;

Didier Fontenille - didier.fontenille@mpl.ird.fr; Laurence Marrama - Imarrama@pasteur-guadeloupe.fr; Alioune B Ly - ly@pasteur.sn;

Fatoumata D Sarr - fdsarr@pasteur.sn; Aïssatou Toure - atoure@pasteur.sn; Jean-François Trape - Jean-Francois.Trape@ird.sn;

André Spiegel - aspiegel@ pasteur-cayenne.fr; Christophe Rogier - christophe.rogier@wanadoo.fr; Pierre Druilhe* - druilhe@pasteur.fr

* Corresponding author
\end{abstract}

Published: 15 July 2009

Malaria Journal 2009, 8:161 doi:10.1186/1475-2875-8-161
Received: 9 June 2009

Accepted: 15 July 2009

This article is available from: http://www.malariajournal.com/content/8/I/16I

(C) 2009 Tall et al; licensee BioMed Central Ltd.

This is an Open Access article distributed under the terms of the Creative Commons Attribution License (http://creativecommons.org/licenses/by/2.0), which permits unrestricted use, distribution, and reproduction in any medium, provided the original work is properly cited.

\begin{abstract}
Background: The time necessary for malaria parasite to re-appear in the blood following treatment (re-infection time) is an indirect method for evaluating the immune defences operating against pre-erythrocytic and early erythrocytic malaria stages. Few longitudinal data are available in populations in whom malaria transmission level had also been measured.
\end{abstract}

Methods: One hundred and ten individuals from the village of Ndiop (Senegal), aged between one and 72 years, were cured of malaria by quinine ( $25 \mathrm{mg} /$ day oral Quinimax ${ }^{\mathrm{TM}}$ in three equal daily doses, for seven days). Thereafter, thick blood films were examined to detect the reappearance of Plasmodium falciparum every week, for II weeks after treatment. Malaria transmission was simultaneously measured weekly by night collection of biting mosquitoes.

Results: Malaria transmission was on average 15.3 infective bites per person during the 77 days follow up. The median reappearance time for the whole study population was 46.8 days, whereas individuals would have received an average one infective bite every 5 days. At the end of the follow-up, after 77 days, 103 of the 110 individuals ( $93.6 \%$; $\mathrm{Cl} 95 \%$ [89.098.2]) had been re-infected with $P$. falciparum. The median reappearance time ('re-positivation') was longer in subjects with patent parasitaemia at enrolment than in parasitologically-negative individuals (58 days vs. $45.9 ; p=0.03$ ) and in adults $>30$ years than in younger subjects ( 58.6 days vs. $42.7 ; \mathrm{p}=0.0002$ ). In a multivariate Cox $\mathrm{PH}$ model controlling for the sickle cell trait, G6PD deficiency and the type of habitat, the presence of parasitaemia at enrolment and age $\geq 30$ years were independently predictive of a reduced risk of re-infection $(\mathrm{PH}=0.5$ [95\% Cl: $0.3-0.9]$ and $0.4 ;$ [95\% Cl: $0.2-$ 0.6] respectively).

Conclusion: Results indicate the existence of a substantial resistance to sporozoites inoculations, but which was ultimately overcome in almost every individual after $2 \mathrm{I} / 2$ months of natural challenges. Such a study design and the results obtained suggest that, despite a small sample size, this approach can contribute to assess the impact of intervention methods, such as the efficacy vector-control measures or of malaria pre-erythrocytic stages vaccines. 


\section{Background}

Exposure to parasites induces immune responses, which can reduce parasite loads. The acquisition of a state of protection against clinical malaria, called premunition, by individuals who are regularly infected with Plasmodium falciparum enables them to control parasite densities to low levels and, thereby, to reduce the incidence of clinical malaria episodes. This control is usually considered to be mainly induced by and effective against erythrocytic forms of $P$. falciparum [1]. However, it has been proposed that the protection acquired by exposure to infection, may also extend to other stages including pre-erythrocytic forms [2]. Experimental studies have shown that exposure to irradiated sporozoites induces parasitological and clinical immunity[2]. In natural conditions, exposure to infected mosquitoe bites induces immune responses to sporozoite surface antigens and their intensity is a function of the Entomological Inoculation Rate (EIR), and, obviously, of age, reflecting the cumulative number of sporozoites received [3-6]. Naturally acquired antibodies also strongly inhibit sporozoite invasion into hepatocytes under in vitro conditions [7].

This exposure-induced immunity may explain why in many hyperendemic areas the incidence of malaria attacks is always markedly lower than predicted by the number of sporozoite inoculations. For instance, 1-5 malaria attacks/child/year are observed in areas where children receive $>100$ infective inocula by mosquitoes per year $[8,9]$, suggesting a reduction of the proportion of inoculum that leads to a blood parasitaemia proportional to the antigenic natural stimulation by pre-erythrocytic stages of the parasites. However, as the evaluation of immunity is based on the detection of blood parasitaemia, it is difficult in observational studies to distinguish the respective effects of immune responses against pre-erythrocytic and erythrocytic stages. Several studies have attempted to document the existence of a pre-erythrocytic "brake" [9], which may result from immunity acquired by exposure to antigens from sporozoites and liver stages. It has been shown for instance that a tenfold decrease in malaria transmission is associated, with only a two-fold decrease in malaria morbidity [9] and little changes in parasitaemia. In the Garki project, it proved impossible to make a transmission model fit field observations, unless a strong density-dependent pre-erythrocytic filter, or brake, was assumed [8].

One way of quantifying this control of new malarial inoculations, is to measure the time of reappearance of blood parasites in subjects whose parasitaemia has been cleared by radical cure and who are exposed to measured numbers of infective mosquitoe bites (called 're-positivation' here), i.e. to measure the relative success of new sporozoite inoculations $[8,10-12]$. This method, which is rec- ommended by WHO in the field to test the efficacy of candidate vaccines, has been previously used [13].

In the present study, the relationships between the reinfection time and several host factors were analysed in a Senegalese community living in the village of Ndiop, an area of seasonal malaria transmission. The aim was to identify the host factors involved in this immune resistance or the factors that could serve as intermediate criteria for the evaluation of intervention methods.

\section{Methods}

\section{Follow-up procedure}

The existing set-up of Ndiop, a village of about 350 inhabitants, located in the Sine-Saloum, in the sahelo-sudanian region of Senegal, was used. In this village, numerous malaria studies, particularly extremely close monitoring of clinical malaria attacks (daily active case detection), drug use and entomological follow-up have been in place since 1993, according a protocol that was identical to that used in the village of Dielmo, Senegal. Thick blood smears were prepared Giemsa-stained, and examined by 1,000× light microscopy using oil immersion. Parasitaemias were scored per microliter of blood by counting the number of asexual parasites per 100 white blood cells, assuming 8,000 blood cells per microliter, and multiplying the parasite count by 80 [14]. Malaria transmission is seasonal from July to October. Malaria vectors are Anopheles arabiensis, Anopheles gambiae and Anopheles funestus [15]. Biological tests, including haemoglobin electrophoresis and tests for glucose-6-phosphate dehydrogenase (G6PD) deficiency, were available for most subjects participating in the study. The type of habitat and bed net use were recorded for each subject. One hundred eighteen volunteers, aged one year or above, were enrolled in the study at the end of the dry season (in August, 1997). During the study period, treatment relied on the administration of quinine chlorhydrate (Quinimax ${ }^{\circledR}$, Sanofi-Synthelabo, France), chosen in view of its efficacy in this area of chloroquine resistance, of its fast effect, and its short half-life. It was administered orally at a dose of $8 \mathrm{mg} / \mathrm{kg}$ under medical supervision (with assessment of proper ingestion) at $8 \mathrm{~h}$ intervals (i.e., $25 \mathrm{mg} / \mathrm{kg} / \mathrm{d}$ ) for $7 \mathrm{~d}$. The very short half-life of quinine has the advantage to avoid the persistence of prophylactic concentrations of the drug in the receivers' blood that could provide artificial protection in the following weeks or months, i.e., avoid introducing a confounding factor as compared to other antimalarial drugs. Since free medical care is available $24 \mathrm{~h} / \mathrm{d}$, selftreatment is known to be most uncommon, as ascertained by systematic detection of anti-malarial drugs [16]. In case of systemic adverse events (including fever, headache, nausea, vomiting and asthaenia), the subject was excluded from the study. After seven days of quinine treatment, the thick blood smears of all the participants 
proved to be negative. Thick blood smears were examined every week for 77 days or until reappearance of a patent asexual $P$. falciparum parasitaemia or loss to follow-up (missing two consecutive thick blood smears). The parasite reappearance time was defined as the number of days between the end of quinine treatment and the detection of $P$. falciparum on blood films.

Informed consent was first obtained at community level. The informed consent of each villager (or that of the parents in the case of children) was orally obtained at the beginning of the study after a thorough explanation of its purpose and was renewed at each stage of the survey. The study design received clearance from the Senegal National Ethics Committee (Dakar, Senegal) [17].

\section{Entomological survey}

Night captures using human bait were conducted indoors and outdoors every Monday and Thursday night during the whole study period. The mosquitoes were captured from 9:00 PM until 7:00 AM by four groups of two collectors (two indoors and two outdoors), with each collector alternatively working and resting for $1 \mathrm{hr}$. The field and laboratory processing have been described previously [15].

\section{Statistical analysis}

Differences in reappearance rate of patent Plasmodium parasitaemia related with epidemiological and genetic variables were tested using log-rank test (bivariate analysis) and Cox model (multivariate analysis). The BMDP 7.0 (Statistical Software, Inc., Los Angeles, CA) and SPIDA (Statistical Computing Laboratory, Eastwood, Australia) software packages were used for analysis. Due to the relative small size of the cohort, results could not be analysed for each age group, but the population was divided in two groups below or above 30 years. This cut-off value was defined by using the Akaike index for age either considered as a continuous variable or for various dichotomous variables (i.e. $1-9,1,10-19,20-30,>30,>40$ years etc), and choosing the best fit [18].

\section{Results}

One hundred eighteen persons were included and treated (114 for seven days and four for six days or less). Of the 114 persons who had received the full course of treatment, four left the village during the first week of followup period and were therefore excluded. A total of $110 \mathrm{sub}-$ jects (46 M/64 F; median age [min-max]: 13.9 [1.2-72]) were monitored during the eleven weeks follow-up period and were included in the analysis. Three individuals missed two consecutive thick blood smears on the $7^{\text {th }}$, $42^{\text {nd }}$ and $57^{\text {th }}$ day respectively. Four individuals (3.6\%) used a non-impregnated bed net. Before treatment, the prevalence of $P$. falciparum asexual blood infection was $21.4 \%$ (6/28) among subjects $\geq 30$ years old versus $10.9 \%$ $(9 / 82)$ in subjects $<30$ years old (NS). The prevalence of patent parasitaemia did not differ according to whether an anti-malarial cure had been received during the year preceding the study or according to the time between the previous treatment and the beginning of the study.

Seven hundred forty (740) malaria vectors were captured during 128 person-night collections on human volunteers during the four-month study period, and their salivary glands examined. The resulting EIR was 15.3 infective bites per person during the 77 days follow up period.

\section{Reappearance time}

Two individuals were infected with Plasmodium ovale: a 13 -year old on day 71 and a 38-year old on day 76. Two other individuals were infected with Plasmodium malariae - two children aged 9 and 11 on day 29 and 70, respectively. The following results only concern $P$. falciparum infections.

The results (Figure 1 and Table 1) show that sporozoite inoculations occurring on average every five days did not each led to a patent parasitaemia. Indeed, it took more than one month to detect parasites reappearing in only $25 \%$ of the exposed population and about 50 days (corresponding to an average 10 infective bites) or only half of the population to become positive again. The median reappearance time for the whole human population was 46.8 days. Nevertheless, at the end of the follow-up, after 77 days, the majority, 103 persons (93.6\%; CI 95\% [89.098.2]) had been re-infected with $P$. falciparum, as defined by: i) the documented clearance of parasitaemia after seven days quinine treatment and; ii) the detection of $P$. falciparum on thick blood smear during the follow-up. Among the seven persons without reappearance of $P$. falciparum blood parasitaemia, five subjects were over 30 years old. Conversely, the shortest reappearance time was seven days and was observed in an 8-years-old child.

\section{Bivariate analysis}

Bivariate analysis led to two main observations: the median reappearance time was found to be significantly longer i) in subjects having a patent $P$. falciparum parasitaemia at the time of enrolment than in parasitologicallynegative individuals, and ii) in adults over 30 years as compared to younger subjects ( 58 days vs. $45.9 ; \mathrm{p}=0.03$ and 58.6 days vs. $42.7 ; \mathrm{p}=0.0002$ respectively) (Figure 1 ). Importantly, other parameters, such as the type of housing, the gender and the G6PD deficiency, were not associated with the time to re-infection, and individuals with the sickle cell trait demonstrated a non-significant trend to an extended time to reappearance (Table 1). 


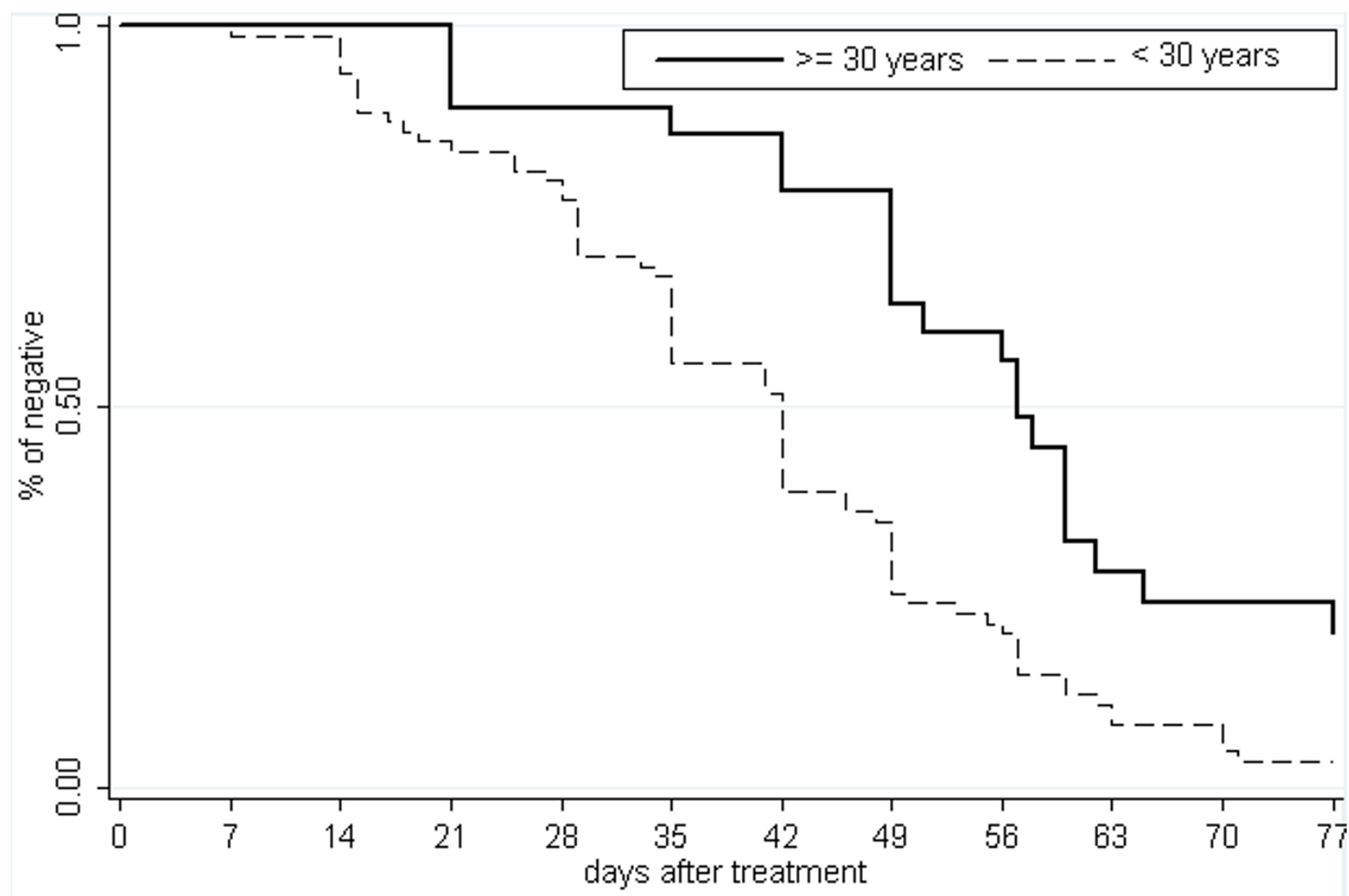

Figure I

Cumulative incidence of asexual Plasmodium falciparum parasitaemia as a function of time after treatment according to age group.

\section{Multivariate analysis}

A multivariate Cox proportional hazards model including age, prior parasitaemia, sickle cell trait, space between the roof and the wall of the house revealed that only age $\geq 30$ years and parasitaemia prior to treatment were independently associated with partial protection from reinfection. The Cox PH was 0.5 [95\% CI: 0.3-0.9] for age (age $<30$ years as reference group) and 0.4 [95\% CI: 0.2-0.6] for prior parasitaemia (clinically non-patent parasitaemia at inclusion as reference group). Using different cut-of values than 30 years also led to conclude about the role of age, but in less significant manner.

\section{Discussion}

In this medium-low transmission area, reinfection studies show a substantial resistance of individuals to sporozoite inoculations. Indeed it required an estimated average of more than six inoculations of sporozoites to positivate 25 percent of the population and more than 10 to positivate half of the cohort. However, after an average of 15 infective bites, all but seven individuals had been undergoing a successful liver schizogony, as shown by patent blood infection. Similar resistance to sporozoite inoculations has already been reported in individuals exposed to both high and low transmission conditions in Senegal, such as in Dielmo, with average 200 infective bites/person/year [19] and in Diohine, with 10 infective bites/person/year [20]. In Dielmo, the median times before reappearance of $P$. falciparum were 22,39 , and 53 days among age-groups $1-6,7-14$, and $\geq 15$ years, respectively, i.e. confirming the strong effect of age, and showing long positivation delays in adults. After 98 days of follow up, corresponding to an average 35 infective bites, $12 \%$ of the population had not been yet become positive again, showing a stronger level of resistance to blood positivation than in Ndiop. In Diohine, the median time to reappearance of $P$. falciparum 
Table I: Reappearance time of asexual P. falciparum parasitaemia according to variables (log-rank test)

\begin{tabular}{|c|c|c|c|c|c|}
\hline & \multicolumn{5}{|c|}{ Reappearance time of $P$. falciparum (days) } \\
\hline & $\mathbf{n}$ & $25 \%$ & $50 \%$ & $75 \%$ & p-value \\
\hline \multicolumn{6}{|c|}{ Parasitaemia before treatment } \\
\hline no & 94 & 33.0 & 45.9 & 57.2 & 0.0338 \\
\hline yes & 15 & 44.1 & 58.0 & 73.9 & \\
\hline \multicolumn{6}{|l|}{ Age } \\
\hline$<30$ years & 82 & 30.7 & 42.7 & 53.5 & 0.0002 \\
\hline$\geq 30$ years & 28 & 50.4 & 58.6 & 68.6 & \\
\hline \multicolumn{6}{|l|}{ Gender } \\
\hline Female & 64 & 33.0 & 48.1 & 58.2 & 0.9646 \\
\hline Male & 46 & 35.0 & 46.4 & 59.8 & \\
\hline \multicolumn{6}{|c|}{ Haemoglobin } \\
\hline AA & 81 & 31.5 & 45.2 & 57.8 & 0.2486 \\
\hline AS & 29 & 40.2 & 51.1 & 61.0 & \\
\hline \multicolumn{6}{|l|}{ G6PD } \\
\hline$<6.9 \mu \mathrm{l}$ & 12 & 35.3 & 48.1 & 58.9 & 0.7743 \\
\hline$\geq 6.9 \mu \mathrm{l}$ & 92 & 31.5 & 45.2 & 59.7 & \\
\hline \multicolumn{6}{|c|}{ Roof of the hut } \\
\hline thatched & 74 & 33.2 & 48.8 & 58.5 & 0.5687 \\
\hline zinc & 36 & 33.6 & 46.5 & 58.9 & \\
\hline \multicolumn{6}{|c|}{ Wall of the hut } \\
\hline cement & 91 & 32.4 & 48.6 & 59.3 & 0.2821 \\
\hline banco & 19 & 36.3 & 44.1 & 57.7 & \\
\hline \multicolumn{6}{|c|}{ Free space between roof and wall } \\
\hline present & 74 & 32.2 & 45.8 & 58.3 & 0.1910 \\
\hline absent & 36 & 34.6 & 49.9 & 60.2 & \\
\hline
\end{tabular}

* values are the number of days until 25 or 50 or $75 \%$ of the studied population was reinfected.

was 28 days in individuals $<40$ years[20]. Similar studies were conducted in the villages of Sotuba (Mali) and in Saradidi (Kenya) respectively, areas of low and high transmission conditions. In Sotuba (Mali), the maximal reinfection rates were observed between 7 and 9 weeks post treatment; 60 of the 88 reinfections occurred during this time period. In these two studies, the analysis was restricted respectively to individuals less than twenty of age and in infants aged from 6 months to 6 years $[21,22]$, whereas in the present study the number of individuals was larger and they belonged to all age groups. In Navrongo (Ghana), where malaria transmission is intense [23], the first infections were identified during week 3 of post-cure follow-up, the majority of new infections occurred during weeks 5-10. Here as well, 98\% had finally become positive again, however only after a long delay of five months of exposure [23]. Results obtained in the very high transmission area of Idete (Tanzania), although gathered by different methods, concur to the same conclusions[24]. The EIR of $1.6(+2.1)$ per person, per day, were much higher than the corresponding blood infection rates. The seasonal increase in EIR (> 4/night) was not accompanied by an increase in new blood infec- tions. Results show that $93 \%$ and $99.6 \%$ of sporozoite inoculations in low and high season respectively, were blocked, i.e. did not result in a detectable blood infection, showing the acquisition of an extremely strong pre-erythrocytic or/and early erythrocytic "brake" under high transmission conditions.

The reappearance time was longer in subjects with parasitaemia upon enrolment than in individuals without, independently of age or administration of antimalarial treatment over the year preceding the study. This result is in apparent contradiction with that recorded in the higher endemic area of Dielmo, where previous parasitaemia was associated with an increased risk of the patient becoming prositive again [19]. However, this observation should be interpreted in the context of differences in anti-blood stage immunity. Indeed, in Ndiop, the clinically non-patent parasitaemia before cure, observed in only 15 individuals, was made of very low parasite densities: $70 \%$ had a parasitaemia of less than eight trophozoites per $\mu$ l. In Dielmo, where transmission is continuous with higher prevalence and higher loads of parasites, the results should be analysed separately for either low or high pre- 
existing parasitaemia. Among the subset of individuals with only low circulating parasitsemia, the reappearance time was also longer, i.e. results are consistent with those observed in Ndiop. In the remaining, with higher initial parasite loads, the susceptibility to sporozoite inoculation increased: an increase of 1,000 trophozoites per $\mu \mathrm{l}$ was significantly associated with an increased risk of positivation by 1.6 [1.2-2.1]. The reasons for this opposite behaviour and effect upon re-positivation are not known $\mathrm{A}$ possible explanation is that the high parasitaemia, which are known to induce an immune suppression[25], decreased acquired defences against the sporozoite stage.

Age was significantly associated with reappearance time in all studies. However, the age at which the risk of reappearance of parasitaemia decreased significantly varied according to the studies and was grossly in relation with transmission intensity, i.e. in relation with exposure to sporozoites and hence potentially to anti-sporozoite immunity. It was significant in individuals $>40$ years in Diohine [20], where the level of transmission is low, in individuals $>30$ years in the present study, and in individuals $>7$ years in Dielmo where the transmission is highest [19]. In Sotuba, the same age trend was observed but it was not significant most likely because of the limited age range of the study population [21]. In other words, individuals acquired faster resistance to reinfection in areas of high transmission, such as Dielmo, than in moderate areas of transmission, such as in Ndiop, or than in areas of low transmission, such as Diohine. This relationship suggests that exposure to higher numbers of sporozoites in areas of high transmission induces a stronger pre-erythrocytic immunity than in areas of moderate or low transmission, i.e. bring support to the concept of sporozoite exposure-dependant immunity. In contrast, the relative success of sporozoite inoculations is maximal in naïve non-immune individuals, as shown by blood parasitaemia induced in volunteers exposed to two infected mosquitoe bites [26], and decreases in malaria-endemic areas, as a function of EIR and age, ie of exposure to sporozoites.

Similar findings have been reported for other stages such as gametocytes, with evidence for gamete-blocking antibodies. Thus exposure to various stages of the parasite life cycle, sporozoite, liver stages, asexual blood stages, gametocytes, would generate a parasite-dependent, stage-specific immunity that modulate the survival of the corresponding stage. The lack of significant effect of the sickle cell trait upon the time it takes for a patient to become positive again may be significant in that respect.

As sickle cell trait is the strongest known factor of protection against erythrocytic stages, this observation brings support to the view that re-positivation studies measures mostly pre-erythrocytic immune defences. Indeed, in the same community, the well-documented strong effect of the sickle cell trait against clinical malaria, including uncomplicated clinical malaria, was confirmed [27], and this effect has been consistently reported elsewhere $[28,29]$. Thus immunity against each stage can be distinguished by distinct features. The sickle cell phenotype does not prevent blood infection, but merely reduces blood parasite densities and thereby prevents symptoms. Indeed, it is well-documented that, in hyperendemic African settings, a majority of individuals carry parasites. However pathological manifestations occur only when parasite densities in the blood are high, a phenomenon which led to define a parasite density threshold to attribute fever to malaria [30,31]. Blood stage anti-parasite immunity prevents symptoms, not infection. In contrast pre-erythrocytic stage immunity prevents infection, regardless of whether the breakthrough parasite reach, or not, a clinical patency threshold of density. Indeed in this, as in previous studies of re-positivation, the emergence of new parasites in the blood did not required treatment in the majority of cases, as pre-existing blood stage immunity controlled parasite loads below the pathogenic threshold. Together this suggests also that AS phenotype or anti-erythrocytic stages immunity modulates parasite densities, whereas pre-erythrocytic immunity modulates the emergence of new blood infections following exposure to sporozoite inoculations. Understandably both types of immunity are related to previous exposure to each stage and hence to age, as described above. The occurrence of clinical malaria corresponds to the lack of ability to control the parasite at each stage, and conversely resistance is likely dependant on the sum of these two immunities. Additional studies are required to better identify the role of each.

\section{Conclusion}

Results obtained in this and in previous studies bring support to the existence of a resistance to sporozoite inoculation which increases, as a function of parasite transmission levels and age, i.e. depending on previous exposure to pre-erythrocytic stages. Further similar studies are required to better delineate the part related to preerythrocytic and to erythrocytic stages immune responses. Indeed, several malaria control measures are targeted against the pre-erythrocytic phase of the cycle, such as vector-control measures, i.e. intra-domiciliary spraying, insecticide-impregnated curtains or bed nets, repellents, and experimental vaccines directed against the sporozoite and/or liver stages, the impact of which would be best evaluated using stage-specific tools. The design and results of re-positivation studies indicate that they can contribute to monitor the efficacy of those interventions.

\section{Competing interests}

The authors declare that they have no competing interests. 


\section{Authors' contributions}

AT responsible for field-implementation, supervision of the team in the field, data collection, analyses, writing of and finalising this paper. CS, DF contributed to entomological protocol design and data collection ABL, FDS, LM supervision of the team in the field, data collection, supported data analyses. AT supported supervision of laboratory team. RP, CR supported writing of this paper. CR established the longitudinal study of the population. PD, JFT, AS contributed to study design, overall supervision of the study, and supported data analysis and writing of this paper. All authors read and approved the final manuscript.

\section{Financial support}

This study was supported by a grant from the Ministère Français de la Coopération et du Développement

\section{Acknowledgements}

We are grateful to the villagers of Ndiop for their active participation and continuing collaboration in the project. We thank Drs El H.T. Mbengue, Ousmane Sarr, N Diagne, F Bâ, Mrs B. Thiam, F. Ndiaye, A. Badiane, J. Faye, EL H. Bâ, P. Ndiaye, G. Ndiaye, A. Ndao, D. Diop, B. Loum, M. Ndiaye, M. Dansokho, M. Cissé, C. Héraut, N. Senghor, B. Diakhaby, for technical support and availability in the field work.

\section{References}

I. Rogier C, Tall A, Diagne N, Fontenille D, Spiegel A, Trape JF: Plasmodium falciparum clinical malaria: lessons from longitudinal studies in Senegal. Parassitologia 1999, 41:255-259.

2. Druilhe P, Renia L, Fidock D: Immunity to liver stages. In Malaria: parasite biology, pathogenesis and protection Edited by: Sherman IW. Washington DC: ASM Press; 1998:5। 3-543.

3. Bottius E, BenMohamed L, Brahimi K, Gras H, Lepers JP, Raharimalala L, Aikawa M, Meis J, Slierendregt B, Tartar A, et al:: A novel Plasmodium falciparum sporozoite and liver stage antigen (SALSA) defines major B, T helper, and CTL epitopes. J Immunol 1996, I 56:2874-2884.

4. Druilhe P, Pradier O, Marc JP, Miltgen F, Mazier D, Parent G: Levels of antibodies to Plasmodium falciparum sporozoite surface antigens reflect malaria transmission rates and are persistent in the absence of reinfection. Infect Immun 1986, 53:393-397.

5. Fidock DA, Bottius E, Brahimi K, Moelans II, Aikawa M, Konings RN, Certa U, Olafsson P, Kaidoh T, Asavanich A, et al.: Cloning and characterization of a novel Plasmodium falciparum sporozoite surface antigen, STARP. Mol Biochem Parasitol 1994 64:219-232

6. Fidock DA, Gras-Masse H, Lepers JP, Brahimi K, Benmohamed L, Mellouk S, Guerin-Marchand C, Londono A, Raharimalala L, Meis JF, et al.: Plasmodium falciparum liver stage antigen- $I$ is well conserved and contains potent $B$ and $\mathbf{T}$ cell determinants. I Immunol 1994, I 53: 1 90-204.

7. Mellouk S, Berbiguier N, Druilhe P, Sedegah M, Galey B, Yuan L, Leef $M$, Charoenvit $Y$, Paul $C$, Hoffman S, et al.: Evaluation of an in vitro assay aimed at measuring protective antibodies against sporozoites. Bull World Health Organ 1990, 68(Suppl):52-59.

8. Molineaux L, Gramiccia G: The Garki Project: Research on the epidemiology and control of malaria in the Sudan savanna of West Africa. Geneva: World Health Organization; 1980.

9. Trape JF, Rogier C: Combating malaria morbidity and mortality by reducing transmission. Parasitol Today 1996, I 2:236-240.

10. Dieye A, Rogier C, Trape JF, Sarthou JL, Druilhe P: HLA class Iassociated resistance to severe malaria: a parasitological reassessment. Parasitol Today 1997, I 3:48-49.
11. Hoffman SL, Oster CN, Plowe CV, Woollett GR, Beier JC, Chulay JD, Wirtz RA, Hollingdale MR, Mugambi M: Naturally acquired antibodies to sporozoites do not prevent malaria: vaccine development implications. Science 1987, 237:639-642.

12. McElroy PD, Beier JC, Oster CN, Onyango FK, Oloo AJ, Lin X, Beadle C, Hoffman SL: Dose- and time-dependent relations between infective Anopheles inoculation and outcomes of Plasmodium falciparum parasitemia among children in western Kenya. Am J Epidemiol 1997, 145:945-956.

13. WHO: Guidelines for the evaluation of Plasmodium falciparum vaccines in populations exposed to natural infection: WHO Geneva TDR/MAL/VAC/97. Word Health Organization; 1997.

14. Rogier C, Ly AB, Tall A, Cisse B, Trape JF: Plasmodium falciparum clinical malaria in Dielmo, a holoendemic area in Senegal: no influence of acquired immunity on initial symptomatology and severity of malaria attacks. Am J Trop Med Hyg 1999, 60:410-420

15. Fontenille D, Lochouarn L, Diatta M, Sokhna C, Dia I, Diagne N Lemasson J], Ba K, Tall A, Rogier C, et al.: Four years' entomological study of the transmission of seasonal malaria in Senegal and the bionomics of Anopheles gambiae and A. arabiensis. Trans R Soc Trop Med Hyg 1997, 91 1:647-652.

16. Trape JF, Rogier C, Konate L, Diagne N, Bouganali H, Canque B, Legros F, Badji A, Ndiaye G, Ndiaye P, et al.: The Dielmo project: a longitudinal study of natural malaria infection and the mechanisms of protective immunity in a community living in a holoendemic area of Senegal. Am J Trop Med Hyg 1994, 51:123-137.

17. Roussilhon C, Oeuvray C, Müller-Graf C, Tall A, Rogier C, Trape JF, Theisen M, Balde A, Pérignon JL, Druilhe P: Long-term clinical protection from falciparum malaria is strongly associated with IgG3 antibodies to merozoite surface protein 3. PLoS Med 2007, 13:e320.

18. Akaike H: A new look to Bayes procedure. Biometrika 1978, 65:53-59.

19. Sokhna CS, Rogier C, Dieye A, Trape JF: Host factors affecting the delay of reappearance of Plasmodium falciparum after radical treatment among a semi-immune population exposed to intense perennial transmission. Am J Trop Med Hyg 2000, 62:266-270.

20. Sokhna CS, Faye FBK, Spiegel A, Dieng H, Trape JF: Rapid reappearance of Plasmodium falciparum after drug treatment among Senegalese adults exposed to moderate seasonal transmission. Am J Trop Med Hyg 200I, 65:167-I70.

21. Sagara I, Sangare D, Dolo G, Guindo A, Sissoko M, Sogoba M, Niambele MB, Yalcoue D, Kaslow DC, Dicko A, et al.: A high malaria reinfection rate in children and young adults living under a low entomological inoculation rate in a periurban area of Bamako, Mali. Am J Trop Med Hyg 2002, 66:310-3I3.

22. Beier JC, Oster CN, Onyango FK, Bales JD, Sherwood JA, Perkins PV, Chumo DK, Koech DV, Whitmire RE, Roberts CR, et al:: Plasmodium falciparum incidence relative to entomologic inoculation rates at a site proposed for testing malaria vaccines in western Kenya. Am J Trop Med Hyg 1994, 50:529-536.

23. Owusu-Agyei S, Koram KA, Baird JK, Utz GC, Binka FN, Nkrumah FK, Fryauff DJ, Hoffman SL: Incidence of symptomatic and asymptomatic Plasmodium falciparum infection following curative therapy in adult residents of northern Ghana. Am J Trop Med Hyg 200I, 65: 197-203.

24. Charlwood JD, Smith T, Lyimo E, Kitua AY, Masanja H, Booth M, Alonso PL, Tanner M: Incidence of Plasmodium falciparum infection in infants in relation to exposure to sporozoite-infected anophelines. Am J Trop Med Hyg 1998, 59:243-25I.

25. Struick S, Riley E: Does malaria suffer from lack of memory? Immunological Reviews 2004, 20 I:268-290.

26. Epstein JE, Rao S, Williams F, Freilich D, Luke T, Sedegah M, de la Vega P, Sacci J, Richie TL, Hoffman SL: Safety and clinical outcome of experimental challenge of human volunteers with Plasmodium falciparum-infected mosquitoes: an update. J Infect Dis 2007, 196:145-154.

27. Spiegel A, Tall A, Raphenon G, Trape JF, Druilhe P: Increased frequency of malaria attacks in subjects co-infected by intestinal worms and Plasmodium falciparum malaria. Trans $R$ Soc Trop Med Hyg 2003, 97(2): 198-199. 
28. Allison AC: Protection afforded by sickle-cell trait against subtertian malareal infection. Br Med J 1954, I:290-294.

29. Marsh K: Immunology of human malaria. In Essential Malariology; London 1990.

30. Trape JF, Peelman P, Morault-Peelman B: Criteria for diagnosing clinical malaria among a semi-immune population exposed to intense and perennial transmission. Trans $R$ Soc Trop Med Hyg 1985, 79:435-442.

31. Rogier C, Commenges D, Trape JF: Evidence for an age-dependent pyrogenic threshold of Plasmodium falciparum parasitemia in highly endemic populations. Am J Trop Med Hyg 1996:613-619.

Publish with Bio Med Central and every scientist can read your work free of charge

"BioMed Central will be the most significant development for disseminating the results of biomedical research in our lifetime. "

Sir Paul Nurse, Cancer Research UK

Your research papers will be:

- available free of charge to the entire biomedical community

- peer reviewed and published immediately upon acceptance

- cited in PubMed and archived on PubMed Central

- yours - you keep the copyright

Submit your manuscript here:

http://www.biomedcentral.com/info/publishing_adv.asp 